\title{
Dépôt d'énergie par rayonnement $X$ dans les gaz rares à haute pression
}

E. Robert, A. Khacef, C. Cachoncinlle et J.M. Pouvesle

GREMI, CNRS, Université d'Orléans, BP. 6759, 45067 Orléans cedex 2, France

\begin{abstract}
Résumé : Nous présentons une modélisation de plasmas de gaz rares excités par des impulsions de rayonnement $X$. L'analyse du dépôt d'énergie montre l'existence de gradients importants de densité d'espèces excitées ou ionisées et souligne l'intérêt de la production de photons $\mathrm{UV}-\mathrm{VUV}$ par flash $\mathrm{X}$. La comparaison modèle expérience permet de confirmer la participation des dimères et trimères ioniques à la fluorescence de l'argon à haute pression sur le domaine $150-300 \mathrm{~nm}$.
\end{abstract}

\section{INTRODUCTION}

Les plasmas de gaz rares à des pressions multi-atmosphériques excités par des impulsions de rayonnement $X$ constituent de nouvelles sources dans le domaine UV-VUV. Le dépôt d'énergie par photons $\mathrm{X}$ d'une dizaine de $\mathrm{keV}$ dans ces milieux purs ou en mélange induit les fluorescences des seconds continus (émission du dimère neutre excité), de continus à plus grandes longueurs d'onde et des ions hétéronucléaires [1]. Le modèle présenté dans ce travail complète les études expérimentales de spectroscopie résolue en temps effectuées pour l'ensemble des gaz rares sur la gamme de pression 0.1-30 bar. La comparaison modèle expérience permet de discuter de l'attribution de la fluorescence des continus à grandes longueurs d'ondes aux différentes espèces ioniques. L'originalité du modèle réside dans la résolution spatiale du dépôt de photons énergétiques dans des milieux à haute pression.

\section{DISCUSSION}

La modélisation est réảlisée sur l'argon dont les spectres ont été très largement étudiés et pour lequel les taux des réactions entre les états neutres ou ionisés de l'atome, du dimère et du trimère sont relativement abondants dans la littérature. Le calcul introduit 18 réactions entre les espèces suivantes: $\mathrm{Ar}^{+}, \mathrm{Ar}^{++}, \mathrm{Ar}_{2}{ }^{+}, \mathrm{Ar}_{2}{ }^{++}, \mathrm{Ar}_{3}{ }^{+}, \mathrm{Ar}_{3}{ }^{++}, \mathrm{Ar}^{*}(4 \mathrm{p}), \mathrm{Ar}^{*}\left(4 \mathrm{~s}^{3} \mathrm{P}_{1}\right), \mathrm{Ar}^{*}\left(4 s^{3} \mathrm{P}_{2}\right), \mathrm{Ar}_{2}{ }^{*}\left(\mathrm{~B}^{\mathrm{l}}\right.$ $\left.\Sigma_{\mathrm{u}}{ }^{+}\right), \mathrm{Ar}_{2}{ }^{*}\left(\mathrm{~A}^{3} \Sigma_{\mathrm{u}}{ }^{+}\right)$. Le programme fournit l'évolution temporelle de la concentration des différentes espèces ainsi que le comportement du maximum de concentration en fonction de la pression sur la gamme 0.1-30 bar.

La figure 1 présente la comparaison à 1.6 bar et 20 bar entre les évolutions temporelles calculées par le modèle et les enregistrements expérimentaux de la fluorescence du second continu à $126 \mathrm{~nm}$. 

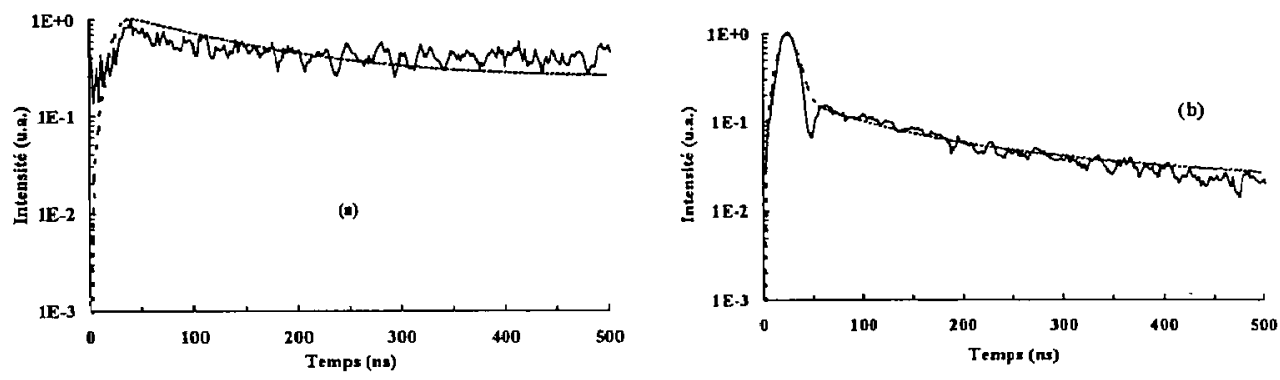

Figure 1: Evolutions temporelles expérimentales et simulées à $126 \mathrm{~nm}$ pour 1.6 bar (a) et 20 bar (b).

La validité du modèle a été testée en comparant les résultats de la simulation aux enregistrements expérimentaux de l'évolution de la fluorescence du second continu. A haute pression, cette fluorescence représente l'étape finale de la relaxation du plasma. Les espèces à son origine sont peuplées tout au long de l'évolution du milieu. De plus, le signal de fluorescence du second continu a une forte dépendance temporelle et en intensité en fonction de la pression.

Le modèle conduit à attribuer les continus de fluorescence, observés à $160,185,200,220$ et $245 \mathrm{~nm}$ en excitation par flash $X$, en partie (aux pressions inférieures à 5 bar) aux dimères ioniques et à plus haute pression aux trimères ioniques de gaz rares. La participation de clusters ioniques déjà évoquée dans les études sur les continus de fluorescence n'avait jusqu'à présent pas été rendue évidente par une confrontation sur un domaine de pression très étendu entre modèle et expérience.

La concordance modèle-expérience confirme la répartition entre l'ionisation et l'excitation au cours de l'interaction photon $\mathrm{X}$-atome de gaz rare. Pour les photons $\mathrm{X}$ générés par notre source $\left(W_{\mathrm{L \alpha}}\right)$ le rapport obtenu est de $70 \%$ d'ionisation et $30 \%$ d'excitation en bon accord avec la littérature [2].

L'analyse, résolue dans l'espace, de la concentration des espèces établit la présence de gradients de concentration le long du chemin de pénétration des rayons $\mathrm{X}$. Cela entraîne des modifications importantes de l'évolution temporelle des fluorescences dont les durées de vie peuvent varier de plusieurs ordres de grandeur. Le calcul de ces gradients pour différents gaz en fonction de la pression, de l'énergie des photons $X$ et de l'épaisseur de plasma rend l'excitation par flash $X$ très prometteuse. $L$ 'excitation de clusters ou cibles solides par notre source $X$ ou de milieux gazeux par des rayons $\mathrm{X}$ plus mous ( $\approx q q e s$ centaines $\mathrm{d}$ 'eV) conduit à la production de plasmas de densité électronique élevée $\left(\approx 10^{16} \mathrm{~cm}^{-3}\right)$ susceptibles d'èmettre très efficacement des photons sur le domaine UV-VUV.

\section{Remerciements.}

Ce travail a été réalisé avec le concours du Conseil Régional du Centre

\section{Références}

[1] Cachoncinlle C., Robert E., Khacef A. et Pouvesle J.M., 'Fluorescence des gaz rares à haute pression excités par flash X rapide', UVX 94, Nouan-le-Fuzelier 13-16 Juin 1994, Annales de physique, 19-Cl, 9 (1994).

[2] J. Bretagne and Y. Louvet, J. Appl. Phys. 61 (3), (1987). 